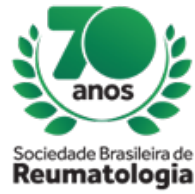

\title{
ABATACEPT: LONG-TERM GAMMA-GLOBULIN AND AUTOANTIBODIES RELATED REDUCTION
}

Valquiria Garcia Dinis (Escola Superior de Ciências da Santa Casa de Misericórdia de Vitória - EMESCAM, Vitória, ES, Brasil), Vilma T Viana (Hospital das Clinicas da Faculdade de Medicina da USP, Sao Paulo, SP, Brasil), Elaine P Leon (Hospital das Clinicas da Faculdade de Medicina da USP, Sao Paulo, SP, Brasil), Clovis A Silva (Instituto da Criança do Hospital das Clinicas da Faculdade de Medicina da USP, Sao Paulo, SP, Brasil), Carla G Saad (Hospital das Clinicas da Faculdade de Medicina da USP, Sao Paulo, SP, Brasil), Julio C Moraes (Hospital das Clinicas da Faculdade de Medicina da USP, Sao Paulo, SP, Brasil), Eloisa Bonfa (Hospital das Clinicas da Faculdade de Medicina da USP, Sao Paulo, SP, Brasil), Ana Cristina Medeiros Ribeiro (Hospital das Clinicas da Faculdade de Medicina da USP, Sao Paulo, SP, Brasil)

\section{BACKGROUND}

Abatacept (ABA) can reduce gamma-globulin levels and specific autoantibodies, but there is no data on anti-vimentin (anti-MCV) and information about anti-cyclic citrullinated peptide (anti-CCP) is conflicting, especially for long-term evaluation. The objective was to assess prospectively the long-term effect on gamma-globulins and autoantibodies of ABA in comparison to tumour necrosis factor inhibitors (TNFi) in rheumatoid arthritis (RA) patients.

\section{MATERIALS AND METHODS}

Eighteen RA patients undergoing abatacept(ABA-RA) and 18 age/sex-matched patients treated with TNFi (TNFi-RA) were compared regarding clinical, inflammatory data, total and specific (IgG, IgM, IgA) gammaglobulins, free light chains (FLC), IgG and IgM rheumatoid Factor (RF), anti-MCV and anti-CCPIII, assessed before and every 6 months, up to 24 months, with a systematic infectious screening protocol. Exclusion criteria: previous abatacept/rituximab or low gamma-globulin $(<0.7 \mathrm{~g} / \mathrm{dL})$.

\section{RESULTS}

At baseline, female sex (78vs.78\%), medians of age (55vs.53years), DAS28 (5.7vs.5.7), levels of gammaglobulin (1.4vs.1.35g/dL), IgG (1,168vs.1,079mg/dL), IgM (107vs.113mg/dL), IgA (333vs.322mg/dL), kappa (342vs.249mg/dL), lambda (170vs.150mg/dL), IgG-RF (63vs.25UI), IgM-RF (76vs.53UI), anti-CCPIII (216vs.189UI), and anti-MCV (202vs.102UI) were comparable ( $p>0.05)$. After 6 months and during the follow up, disease activity parameters improved similarly in both groups ( $p>0.05)$. In ABA-RA, at 6 months, remarkable decreases were observed in total gamma-globulin (1.4vs.1.05g/dL), IgG (1,168vs.997), IgA (333vs. $278 \mathrm{mg} / \mathrm{dL}$ ), kappa (342vs.257mg/dL), lambda (170vs.144mg/dL), IgG-RF (63vs.24UI), IgM-RF (76vs.37UI), anti-CCPIII (216vs.183UI), and anti-MCV (202vs.65UI) in comparison to baseline $(p<0.05)$ and in contrast to TNFi-RA which showed no decrease in any of these parameters $(p>0.05)$, despite of similar disease activity control. Longitudinal measurements in ABA-RA demonstrated that levels of immunoglobulins remained mostly stable for subsequent evaluations, up to 24 months ( $p>0.05$ ), but ABARA had more often transient IgG levels under the lower limit of normality $(66.7 \% \mathrm{vs} .33 .3 \%, \mathrm{p}=0.046)$ and one patient had an isolated lgG level $<600 \mathrm{mg} / \mathrm{dL}$. No severe infection occurred. Regarding autoantibodies, further longitudinal reductions were seen in the 12th month for IgM-RF and in the 18th month for anti$\mathrm{MCV}$, which was lost by 24 months. Despite the decrease in autoantibodies titles, significant changes in sorologic status (immunologic remission) were not observed. Of note, only in ABA-RA group, patients who achieved low disease activity (DAS<3.2) after 6 months $(n=6 ; 33 \%)$ had higher baseline levels of IgG$\operatorname{RF}[208(70-653) v s .25(5-120) ; p=0.003]$ and IgM-RF[143(108-807)vs.20(2-142); $p=0.006]$ compared to those who did not.

\section{CONCLUSION}


These comparative data demonstrate that $A B A$ induces a long-term and non-progressive reduction in gamma-globulin and autoantibodies levels, including anti-CCPIII and anti-MCV, regardless of disease activity control, but not associated with increased infection rate or disappearance of serologic status. 